\title{
Incidência de cólica no lactente e fatores associados: um estudo de coorte
}

\author{
Infantile colic incidence and associated risk factors: a cohort study \\ Maria A.L. Saavedra ${ }^{1}$, Juvenal S. Dias da Costa $^{2}$, Gilberto Garcias ${ }^{3}$, \\ Bernardo L. Horta ${ }^{4}$, Elaine Tomasi ${ }^{5}$, Rodrigo Mendonça ${ }^{6}$
}

\begin{abstract}
Resumo
Objetivos: estabelecer a incidência de cólica no lactente e seus determinantes.

Métodos: entre maio e julho de 1999, a equipe de pesquisa visitou, diariamente, as três principais maternidades da cidade de Pelotas, RS, e todas as mulheres, após o parto, foram entrevistadas, e seus filhos acompanhados aos três meses. Definiu-se a criança com cólica conforme proposto por Wessel. Os possíveis fatores de risco avaliados foram: classe social, escolaridade materna, idade dos pais, tipo e tempo de relacionamento do casal, alterações no relacionamento na gestação, tipo de parto, história reprodutiva, qualidade do pré-natal, experiência anterior de aborto, natimorto ou recém-nascido prévio doente, sexo e tipo de alimentação do lactente. Realizouse o teste do qui-quadrado para comparações entre proporções e análise multivariada através de regressão logística não condicional.

Resultados: conseguiu-se acompanhar 1.086 crianças das 1.195 identificadas. Na visita aos três meses, a incidência de cólica referida pela mãe foi de $80,1 \%$. Entretanto, apenas $16,3 \%$ tinham apresentado cólica de acordo com os critérios de Wessel. Após a análise multivariada, as associações que permaneceram no modelo com desfecho de cólica foram: escolaridade materna, cesariana, idade paterna e amamentação. Mesmo após ajuste para fatores de confusão, as crianças desmamadas tiveram uma chance 1,86 vez maior de ter cólica do que aquelas ainda amamentadas (IC 95\% 1,25-2,77).
\end{abstract}

Conclusão: a maioria das mães não reconheceu corretamente a ocorrência de cólica de acordo com os critérios adotados. O aleitamento materno foi o principal fator de proteção contra o desenvolvimento de cólica.

J Pediatr (Rio J) 2003;79(2):115-22: cólica, choro, relações mãe-filho, aleitamento materno.

1. Mestre em Saúde e Comportamento, UCPel.

2. Mestre em Epidemiologia, UFPel.

3. Doutor em Genética e Biologia Molecular, UFRGS.

4. Doutor em Epidemiologia, McGill.

5. Mestre em Epidemiologia, UFPel.

6. Acadêmico da Faculdade de Medicina, UCPel.

Artigo submetido em 21.03.02, aceito em 22.01.03.

\begin{abstract}
Objective: to assess the incidence of infantile colic and its determinants.

Methods: between May and July 1999, the research team performed daily visits to the three main maternities of Pelotas. All women were interviewed after the delivery and their infants were targeted for follow-up at three months. Colic was defined according to Wessel. The following risk factors were evaluated: social class, mother's educational level, parents' age, parents' divorce, changes in the parents' relationship during pregnancy, type of delivery, reproductive history, quality of prenatal care, previous abortion, previous stillbirths, previous newborn with health problems, gender, and infant's feeding pattern. Chi-square test was used to compare proportions and conditional logistic regression was applied in the multivariate model.

Results: we managed to follow 1,086 of the 1,195 infants. At the three months visit, $80.1 \%$ of the mothers reported that their infants had had colic. On the other hand, only $16.3 \%$ was considered as having colic according to Wessel criteria. Mother's educational level, father's age, type of delivery and breast-feeding duration remained in the multivariate model. Even after controlling for possible-confounding factors, the chances of having colic was 1.86 times higher among non-breastfed infants than among breastfed infants.
\end{abstract}

Conclusion: most mothers misclassify the occurrence of colic. Breastfeeding is the main protective factor.

J Pediatr (Rio J) 2003;79(2):115-22: colic, crying, motherchild relationship, breastfeeding.

\section{Introdução}

A definição clínica para cólica do lactente mais aceita na literatura é a de Wessel ${ }^{1}$, que descreve esta síndrome como paroxismos de irritabilidade, agitação ou choro, durante pelo menos três horas por dia, mais de três dias na semana 
em pelo menos três semanas, em crianças saudáveis. O lactente chora de forma inconsolável, geralmente ao anoitecer, sem uma causa identificável e com exame físico normal. Esse problema usualmente surge na segunda semana de vida, intensifica-se entre a quarta e a sexta semana e gradativamente alivia, desaparecendo até o terceiro mês de vida.

A cólica do lactente é uma condição transitória, sem riscos de mortalidade e que não interfere no crescimento da criança. Entretanto, além de ser uma situação extremamente estressante para a família e para o pediatra, pode alterar o desenvolvimento pelo reflexo negativo na interação da criança com seus pais e, ainda, deixar seqüelas emocionais, levando ao surgimento de transtornos somáticos no lactente $^{2}$. Estudos prospectivos têm demonstrado que a cólica produz sensação de incompetência nos pais, discórdia entre o casal e aumenta o risco de abuso e violência doméstica ${ }^{2,3}$.

O choro do lactente é, de todos os padrões de comportamento pré-verbais, o que mais chama a atenção dos pais. Na tentativa de reconhecer o padrão de choro em diferentes circunstâncias como fome, dor e frio e aperfeiçoar a definição de cólica, vários trabalhos com gravações, observações diárias e análises do espectro do choro foram realizados. Demonstrou-se a capacidade das mães reconhecerem os padrões de choro de seus filhos, parecendo ser confiável esta informação a partir da percepção materna, e o choro seria o critério mais fidedigno para o diagnóstico de cóli$\mathrm{ca}^{4-7}$.

No que diz respeito à sua etiologia, do ponto de vista da gastrenterologia, já foram sugeridos: imaturidade ou alergia gastrintestinal, intolerância ao leite de vaca, má absorção e refluxo gastresofágico ${ }^{8.9}$. Reforçando a hipótese de que a cólica pode ser decorrente da intolerância ao leite de vaca, o tratamento que utiliza leite de soja ou fórmulas hipoalergênicas mostra redução dos sintomas ${ }^{10,11}$.

Alguns autores também apontam para a importância da relação familiar. Forsyth et al. ${ }^{12}$ citam preocupações em relação à alimentação e o baixo nível de educação materna como fatores associados à cólica. Para outros autores, a cólica pode ser um sintoma de disfunção na díade mãe-filho e/ou desta no âmbito familiar2,13,14. O ambiente se caracterizaria por uma manipulação inadequada devido à inexperiência, ansiedade, depressão ou raiva dos pais. Experiências de sintomas clínicos, descontentamento com a relação sexual na gravidez e uma vivência negativa no parto também têm sido associados à ocorrência de cólica, assim como isolamento social na gestação e uma mãe insegura na ocasião do nascimento ${ }^{15}$. Por outro lado, trabalhos recentes sugerem a cólica como uma manifestação do desenvolvimento emocional normal, como uma menor capacidade do lactente em regular a duração de seu choro, ou que seja uma questão de temperamento ${ }^{16,17}$.

A melhora do quadro através de condutas terapêuticas que modificam o comportamento das mães em relação às crianças (tais como não deixar o bebê chorar, dispositivos de embalo ou vibração mecânica, formas de pegar ao colo) apóiam a hipótese de causas extrínsecas para a cóli$\mathrm{ca}^{3,5,9,18,19}$. Entretanto, alguns trabalhos sobre o tema foram conduzidos em populações altamente selecionadas, o que dificulta a sua generalização.

Com o intuito de se quantificar a magnitude do problema e os seus determinantes, de forma a fornecer também dados que possam aprimorar a habilidade dos pediatras na identificação de quais pais têm maior possibilidade de seus filhos desenvolverem cólica, e na tentativa de se prevenir outros transtornos psicossomáticos, realizou-se um estudo de coorte na cidade de Pelotas, RS.

\section{Métodos}

Este estudo de coorte inicialmente identificou todas as crianças nascidas vivas em três maternidades da cidade (onde ocorrem cerca de 99\% dos nascimentos) e cujas mães residiam na zona urbana de Pelotas, Rio Grande do Sul, no período de maio a julho de 1999. As mães, no período pósparto imediato, foram convidadas a responder um questionário padronizado e pré-codificado. Foram excluídas as mães que não estavam em alojamento conjunto por patologias (ou da mãe ou do recém-nascido).

Calculou-se o tamanho da amostra estimando-se a incidência de cólica do lactente em torno de $30 \%$, uma margem de erro de $3 \%$ e com nível de confiança de $95 \%$. Adicionouse $20 \%$ para possíveis perdas, e assim o tamanho da amostra foi de 1.100 crianças.

As seguintes variáveis foram avaliadas: características demográficas dos pais (idade, tipo e anos de união, história reprodutiva) e perfil da relação familiar (tipo e alteração no relacionamento entre o casal durante a gestação, reação paterna à notícia da gravidez, alteração no relacionamento sexual durante a gestação, como a mãe considerou o apoio do parceiro e da família à gestação). A qualidade do prénatal foi avaliada conforme Kessner, modificado por Take$\mathrm{da}^{20}$, que combina o número de consultas e o período de início do pré-natal. Esse critério considera a gestante com mais de cinco consultas, e a primeira até o quarto mês, como tendo um pré-natal adequado; as mães que iniciam após o sétimo mês de gravidez e fazem menos de quatro consultas teriam um pré-natal inadequado. As demais são classificadas como pré-natal intermediário.

Avaliou-se também sexo e peso de nascimento da criança, intercorrências clínicas na gestação atual, experiência materna anterior com aborto, natimorto ou neonato doente, aceitação da auto-imagem como grávida, tipo de parto, uso de algum tipo de indução ao trabalho de parto, expectativa e cansaço em relação ao parto e classe social, de acordo com a classificação proposta pela Associação Brasileira de Institutos de Pesquisa de Mercado, que utiliza um escore pontuando bens materiais adquiridos, escolaridade, condições sanitárias e número de empregados domésticos; e a partir deste escore, categoriza cinco classes ${ }^{21}$. 
Quando as crianças avaliadas no estudo perinatal completaram três meses, realizou-se uma visita domiciliar e aplicou-se um questionário padronizado e pré-codificado às mães, buscando-se informações sobre a saúde da criança. Definiu-se a criança com cólica como aquela cuja mãe relatou que chorava mais de três horas por dia, mais de três dias na semana, durante pelo menos três semanas. Verificou-se também a ocorrência de fatores que poderiam estar associados a choro excessivo e que poderiam ser confundidos com cólica (otite média, fraturas, infecção urinária). Essas informações foram investigadas em relação a cada um aos três meses de idade. Após as variáveis indicativas de saúde da criança e da mãe, apresentou-se as variáveis que avaliaram o número de horas de choro do lactente. Assim, as mães desconheciam o objeto do estudo. Após as questões referentes à alimentação, a mãe era questionada se seu filho tinha, ou já havia tido, cólicas, aceitando-se a sua percepção.

Estudantes de medicina realizaram todas as entrevistas, sendo previamente treinados quanto à forma de aplicação e preenchimento do questionário. Por haver perguntas de caráter mais íntimo, nas maternidades, utilizou-se apenas entrevistadoras do sexo feminino, as quais desconheciam o objetivo do estudo.

Os próprios entrevistadores codificaram os questionários, e posteriormente a coordenação do estudo fez a revisão da codificação e a classificação das questões abertas. Semanalmente ocorreram reuniões com o objetivo de se aperfeiçoar a coleta de dados e recebimento do material. Para controle de qualidade, os supervisores repetiram cerca de $5 \%$ das entrevistas de forma aleatória.

Através do programa Epi-Info 6.0, versão 6.02, criou-se o banco de dados. Digitaram-se os dados duas vezes e comparou-se no Validate, na tentativa de se evitar os erros de digitação. Para análise estatística, utilizou-se o programa SPSS 10.0 for Windows, assim como o teste do quiquadrado, para comparações entre proporções. Na análise multivariada, através de regressão logística não condicional, introduziu-se as variáveis conforme níveis de causalidade do modelo hierárquico. O primeiro nível incluiu as variáveis socioeconômicas e demográficas, o segundo, relacionamento familiar e eventos relacionados à gestação, e o terceiro, características do parto e alimentação do lactente. Para cada nível, realizou-se uma equação de regressão por eliminação retrógrada, descartando-se todas as variáveis com $p>0,20$. As variáveis que permaneceram no primeiro nível foram incluídas na equação do segundo nível, sendo novamente eliminadas aquelas deste nível com $\mathrm{p}>0,20$. Este procedimento foi repetido para o terceiro nível. O nível "p" de 0,20 foi escolhido, pois variáveis de confusão podem afetar as estimativas, mesmo quando seu nível de significância não alcança 0,05 . Para a apresentação dos resultados, considerou-se como significativas as variáveis com "p" igual ou inferior a 0,05 .

\section{Resultados}

Inicialmente 1.195 mães responderam ao primeiro questionário e, destes, 1.086 crianças foram visitadas aos três meses. Das $109(9,1 \%)$ crianças que não foram acompanhadas, 28 tinham falecido, duas haviam sido abandonadas pelos pais, uma tinha os pais surdos-mudos e as demais não foram localizadas. Não houve diferença entre as crianças acompanhadas ou não, no que diz respeito à idade materna, pré-natal e tipo de parto.

Aceitando-se a percepção materna para a ocorrência de cólica, 870 mães $(80,1 \%)$ responderam que seus filhos, no terceiro mês de vida, tinham ou já haviam tido cólica. Entretanto, apenas 177 crianças (16,3\%) tiveram cólica de acordo com o critério temporal de choro de Wessel.

A Tabela 1 apresenta as características socioeconômicas e demográficas da população estudada, na qual se observa que cerca de $60 \%$ das crianças pertenciam às classes sociais D e E. Quando nasceram, cerca de uma em cada cinco crianças não tinha os pais vivendo juntos. Quanto à escolaridade materna, apenas $18 \%$ das mães completaram 11 ou mais anos de estudo, e 59\% eram analfabetas ou tinham menos de 3 anos de estudo.

A incidência de cólica foi independente do sexo, peso de nascimento e classe social da criança. Chama a atenção uma maior incidência em mães divorciadas, desquitadas ou viúvas, entretanto esta categoria não mostrou uma associação estatisticamente significativa (Tabela 1).

No que concerne à qualidade do pré-natal, a maioria das mães $(73,1 \%)$ tiveram um pré-natal considerado como adequado. Cerca de um terço das mães eram primigestas (Tabela 2). O início da amamentação foi quase universal, mas a sua duração foi baixa, uma vez que apenas $62,5 \%$ das crianças ainda eram amamentadas aos três meses (Tabela $3)$.

As variáveis que avaliaram o relacionamento dos pais, alterações na vida sexual, apoio da família durante a gestação não apresentaram associação com a incidência de cólica nos três primeiros meses de vida. Do mesmo modo, para fatores como a qualidade do pré-natal, mães primigestas, tipos de parto, experiências anteriores de aborto ou natimorto, não foram encontradas diferenças significativas, conforme mostra a Tabela 2.

A Figura 1 apresenta o modelo hierárquico final, ou seja, aquelas variáveis que permaneceram no modelo de análise multivariada. Surpreendentemente, a chance de cólica nas crianças cujas mães têm maior escolaridade foi menor. A maioria dos pais $(81,7 \%)$ tinha entre 20 e 39 anos de idade, e a incidência de cólica esteve inversamente relacionada com a idade do pai, assim a chance de ter cólica foi 0,43 vez maior (IC 95 0,20-0,93) entre as crianças com pais maiores de 40 anos em relação aquelas com pais com idade entre 20 e 29 anos.

Em relação à experiência anterior de doença com o recém-nascido, o efeito negativo de tal experiência aumen- 
Tabela 1 - Presença de cólicas, segundo critério temporal de choro, em relação às variáveis socioeconômicas e demográficas - Pelotas, 1999

\begin{tabular}{|c|c|c|c|c|}
\hline Variáveis & $\mathbf{N}^{\mathbf{o}}$ & $\begin{array}{c}\text { Incidência } \\
\text { de cólica }(\%)\end{array}$ & $\begin{array}{c}\text { Razão } \\
\text { de chance }\end{array}$ & $\begin{array}{c}\text { Intervalo de } \\
\text { confiança }(95 \%)\end{array}$ \\
\hline \multicolumn{5}{|l|}{ Classe social } \\
\hline Classe A e Classe B & 133 & 12,2 & 1,0 & \\
\hline Classe C & 341 & 16,1 & 1,37 & $0,74-2,56$ \\
\hline Classe D & 462 & 16,3 & 1,40 & $0,77-2,55$ \\
\hline Classe E & 256 & 19,2 & 1,70 & $0,90-3,22$ \\
\hline \multicolumn{5}{|l|}{ Sexo } \\
\hline Masculino & 578 & 16,7 & 1,0 & \\
\hline Feminino & 614 & 16,0 & 0,94 & $0,68-1,30$ \\
\hline \multicolumn{5}{|l|}{ Peso ao nascer } \\
\hline Menos de $2.500 \mathrm{~g}$ & 98 & 14,3 & 1,0 & \\
\hline $2.500 \mathrm{~g}-4.500 \mathrm{~g}$ & 1.085 & 16,6 & 1,19 & $0,14-9,97$ \\
\hline Mais de $4.500 \mathrm{~g}$ & 8 & 14,0 & 14,0 & $0,10-8,80$ \\
\hline \multicolumn{5}{|l|}{ Idade materna } \\
\hline Menor ou igual a 19 anos & 208 & 14,9 & 1,0 & \\
\hline De 20 a 35 anos & 864 & 17,1 & 1,17 & $0,75-1.82$ \\
\hline Maior de 35 anos & 120 & 14,0 & 0,93 & $0,48-1,81$ \\
\hline \multicolumn{5}{|l|}{ Anos de escolaridade materna } \\
\hline Maior ou igual a 11 anos & 215 & 17,3 & 1 & \\
\hline De 8 a 10 anos & 273 & 14,2 & 0,55 & $0,30-0,99$ \\
\hline De 4 a 7 anos & 545 & 16,6 & 0,92 & $0,57-1,49$ \\
\hline De zero a 3 anos & 159 & 18,2 & 0,77 & $0,39-1,54$ \\
\hline \multicolumn{5}{|l|}{ Tipo de união do casal } \\
\hline Casada & 401 & 16,7 & 1,0 & \\
\hline Em união & 592 & 15,5 & 0,91 & $0,64-1,31$ \\
\hline Divorciada/desquitada/viúva & 17 & 31,3 & 2,27 & $0,76-6,76$ \\
\hline Solteira & 182 & 16,9 & 1,01 & $0,61-1,66$ \\
\hline \multicolumn{5}{|l|}{ Tempo de união do casal } \\
\hline Até doze meses & 144 & 12,9 & 1,0 & \\
\hline De 1 ano e 1 mês a 2 anos & 119 & 15,5 & 1,24 & $0,59-2,60$ \\
\hline De 2 anos e 1 mês a 5 anos & 277 & 17,8 & 1,46 & $0,80-2,68$ \\
\hline De 5 anos e 1 mês a 10 anos & 267 & 17,4 & 1,42 & $0,77-2,61$ \\
\hline Mais de 10 anos & 165 & 12,3 & 0,95 & $0,47-1,91$ \\
\hline Não estão mais juntos & 218 & 18,0 & 1,48 & $0,79-2,78$ \\
\hline
\end{tabular}

tou, mas o seu intervalo de confiança continuou a incluir a unidade e, conseqüentemente, a associação não foi estatisticamente significativa, o mesmo ocorrendo para um parto considerado pela mãe como traumático.

Mesmo após ajuste para possíveis fatores de confusão, a chance de ter cólica foi 1,86 (IC 95\% 1,25-2,77) vezes maior entre as crianças que haviam deixado de receber leite materno do que entre as que ainda eram amamentadas. As crianças que nasceram de cesariana também apresentaram uma maior chance de ter cólica (R0 1,55 - IC95\% 1,042,32).

\section{Discussão}

A cólica do lactente é uma síndrome bastante subjetiva, com achados contraditórios, e há muitos estudos com problemas metodológicos, como, por exemplo, amostras não representativas da população, diferentes definições para cólica, conforme mostra uma recente meta-análise ${ }^{10}$. Uma vez que os estudos de coorte têm como principais vantagens a possibilidade de avaliar a incidência da doença e seus fatores de risco, não sendo suscetíveis ao viés de causalidade reversa, este foi o delineamento escolhido. Além disso, as perdas de acompanhamento foram baixas, portanto nos- 
Tabela 2 - Presença de cólicas do lactente, segundo critério temporal de choro, em relação a variáveis prénatais - Pelotas, 1999

\begin{tabular}{|c|c|c|c|c|}
\hline Variáveis & $\mathbf{N}^{\mathbf{o}}$ & $\begin{array}{c}\text { Incidência } \\
\text { de cólica }(\%)\end{array}$ & $\begin{array}{c}\text { Razão } \\
\text { de chance }\end{array}$ & $\begin{array}{c}\text { Intervalo de } \\
\text { confiança }(95 \%)\end{array}$ \\
\hline \multicolumn{5}{|l|}{ Pré-natal } \\
\hline Adequado & 874 & 15,9 & 1,0 & \\
\hline Intermediário & 250 & 15,3 & 0,95 & $0,62-1,44$ \\
\hline Inadequado & 46 & 21,6 & 1,45 & $0,65-3,25$ \\
\hline \multicolumn{5}{|c|}{ Número de gestações } \\
\hline Uma & 416 & 16,8 & 1,0 & \\
\hline Duas & 304 & 19,1 & 1,17 & $0,55-1,20$ \\
\hline Três ou mais & 472 & 16,8 & 0,81 & $0,78-1,75$ \\
\hline \multicolumn{5}{|c|}{ Número de filhos vivos } \\
\hline Um & 474 & 17,7 & 1,0 & \\
\hline Dois & 317 & 14,3 & 0,77 & $0,51-1,17$ \\
\hline Três ou mais & 401 & 16,0 & 0,91 & $0,62-1,32$ \\
\hline \multicolumn{5}{|l|}{ Tipo de parto } \\
\hline Normal & 704 & 15,3 & 1,0 & \\
\hline Cesariana & 379 & 18,2 & 1,55 & $1,04-2,32$ \\
\hline \multicolumn{5}{|c|}{ Experiência anterior de natimorto } \\
\hline Não & 1.168 & 16,3 & 1,0 & \\
\hline Sim & 23 & 20,0 & 1,29 & $0,42-3,89$ \\
\hline \multicolumn{5}{|c|}{ Número de abortos prévios } \\
\hline Nenhum & 1.013 & 16,6 & 1,0 & \\
\hline Um & 147 & 14,4 & 0,84 & $0,50-1,41$ \\
\hline Dois ou mais & 33 & 15,6 & 0,92 & $0,35-2,44$ \\
\hline \multicolumn{5}{|c|}{ Ameaça de aborto nesta gestação } \\
\hline Não & 1.067 & 16,0 & 1,0 & \\
\hline Sim & 126 & 19,3 & 1,25 & $0,75-2,07$ \\
\hline
\end{tabular}

so estudo não é suscetível ao viés de seleção, o que pode ocorrer neste tipo de estudo. Finalmente, definimos a ocorrência de cólica a partir dos critérios propostos por Wes$\mathrm{sel}^{1}$, que é o critério diagnóstico de cólica em lactentes que tem sido mais aceito ${ }^{10,19}$.
A inexistência de uma definição padronizada para cólica do lactente torna difícil a determinação da sua magnitude. Encontrou-se na literatura que em torno de 10 a $50 \%$ dos lactentes sadios e bem alimentados apresentavam cólicas $^{10,19}$. A heterogeneidade de conceitos, metodologia e

Tabela 3 - Presença de cólicas do lactente, segundo critério temporal de choro, em relação à amamentação Pelotas, 1999

\begin{tabular}{|c|c|c|c|c|}
\hline Variáveis & $\mathbf{N}^{\mathbf{o}}$ & $\begin{array}{c}\text { Incidência } \\
\text { de cólica (\%) }\end{array}$ & $\begin{array}{c}\text { Razão } \\
\text { de chance }\end{array}$ & $\begin{array}{c}\text { Intervalo de } \\
\text { confiança }(95 \%)\end{array}$ \\
\hline \multicolumn{5}{|c|}{ Amamentação no $3^{\circ}$ mês } \\
\hline Está mamando & 679 & 14,0 & 1,0 & \\
\hline Deixou de mamar & 362 & 21,0 & 1,63 & $1,16-2,27$ \\
\hline Nunca mamou & 45 & 13,3 & 0,94 & $0,38-2,29$ \\
\hline \multicolumn{5}{|c|}{ Tempo que recebeu seio materno } \\
\hline Nunca mamou & 45 & 13,3 & 0,94 & $0,38-2,29$ \\
\hline 1 a 7 dias & 30 & 13,3 & 0,94 & $0,32-2,76$ \\
\hline 8 a 30 dias & 141 & 24,8 & 2,02 & $1,30-3,14$ \\
\hline 31 a 60 dias & 134 & 19,4 & 1,47 & \\
\hline De 61 dias a 90 dias & 57 & 19,3 & 1,46 & $0,91-2,38$ \\
\hline Ainda mama & 678 & 14,0 & 1,0 & $0,73-2,93$ \\
\hline
\end{tabular}


tipos de estudos realizados reflete esta diferença na incidência $^{19}$. No Rio Grande do Sul, em 1987, 84,3\% dos pediatras tinham esta queixa como um problema freqüente na sua prática clínica, usando como critério diagnóstico o choro persistente e inconsolável ${ }^{22}$. Em nosso estudo, a incidência cumulativa de cólica referida pela mãe foi de $80,1 \%$. Porém a incidência cumulativa, estabelecida através dos critérios de Wessel, foi de 16,3\%, demonstrando que a cólica tem uma magnitude considerável, mas não com a freqüência que a população identifica e contrariando estudos que aceitam a percepção materna ${ }^{4,5,23}$.

A associação entre o desenvolvimento de cólica e fatores como a idade da mãe, tipo de união, ordem de nascimento, peso ao nascer e classe social não mostraram diferenças significativas, assim como não haviam sido evidenciadas na revisão de Lucassen $^{19}$, e contrariando o estudo de Crowcroft ${ }^{24}$.

Não se encontrou outros estudos que relatem a existência de uma associação entre cesariana e cólica. Como o valor do limite inferior do intervalo de confiança é baixo, não podemos descartar totalmente o acaso como uma das explicações para este achado. Uma vivência de parto considerada pelos pais como traumática foi descrito por Rau- tava ${ }^{15}$ como fator associado ao desenvolvimento de cólica, mas nem toda cesárea pode ser classificada como traumática, e, para as mães que responderam que seu parto foi pior do que esperavam, o intervalo de confiança continuou englobando a unidade, descartando este fator como associado ao desfecho da presença de cólica.

Contrariando o relatado por Rautava ${ }^{15}$, não observamos uma maior chance de ocorrência de cólica nas crianças cujos pais relataram uma má adaptação à gestação, mudanças negativas no relacionamento sexual do casal e doenças durante a gravidez.

Ao se analisar a presente associação entre amamentação e o desenvolvimento de cólica, a causalidade reversa é um importante viés, pois as mães de crianças que choram mais podem interromper a amamentação por relacionarem a presença desta condição ao tipo de alimentação, ou acharem que o seu leite é insuficiente ou fraco ${ }^{25-27}$. Para se prevenir este viés, considerou-se como amamentadas aquelas crianças que ainda recebiam leite materno no momento em que começaram a ter cólicas. Esta análise persistiu revelando significância, demonstrando uma associação efetiva entre desmame precoce e a presença de cólica. Este achado contraria outros ${ }^{15,24-27}$ que comparam o aleitamen-

\begin{tabular}{|llll|}
\hline \multicolumn{2}{|c}{ Escolaridade da mãe (anos) } & \multicolumn{2}{c|}{ Idade do pai (anos) } \\
$\leq 3$ & $0,77(0,39-1,54)$ & $<20$ & $1,25(0,55-2,84)$ \\
$4-7$ & $0,92(0,57-1,49)$ & $20-29$ & Referência \\
$8-10$ & $0,55(0,30-0,99)$ & $30-39$ & $0,91(0,61-1,36)$ \\
$\geq 11$ & Referência & $\geq 40$ & $0,43(0,20-0,93)$ \\
\hline
\end{tabular}

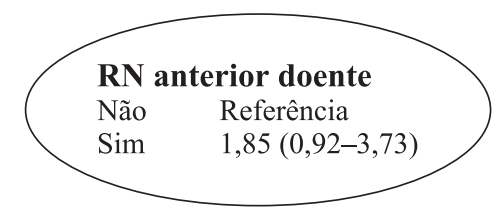

\begin{tabular}{ll}
\multicolumn{2}{l}{ Amamentação aos 3 meses } \\
Ainda mama & Referência \\
Não mama mais & $1,86(1,25-2,77)$ \\
Nunca mamou & $1,60(0,57-4,47)$
\end{tabular}

Tipo de parto

Normal Referência

Cesariana $1,55(1,04-2,32)$

\begin{tabular}{ll}
\multicolumn{2}{l}{ Expectativa do parto } \\
Foi melhor & Referência \\
Como esperava & $0,52(0,24-1,17)$ \\
Foi pior & $1,26(0,84-1,90)$ \\
Não sabe dizer & $0,35(0,08-1,52)$
\end{tabular}

Figura 1 - Modelo hierárquico final para o desfecho de cólica do lactente - Pelotas, 1999 
to materno com alimentação por mamadeira, entretanto, no estudo de Crowcroft ${ }^{24}$, a razão de chance encontrada foi de apenas 1,09 e, em Recife, o estudo de Sarinho e colaboradores $^{26}$ também mostrou que as crianças amamentadas exclusivamente ao seio materno tinham uma chance cerca de três vezes menor de apresentar cólica em relação às crianças desmamadas, mas, devido ao pequeno tamanho da amostra, a associação não foi estatisticamente significativa. $\mathrm{Na}$ década de 50, Wessel apresentou o conceito mais aceito para o problema, mas não identificou nenhuma correlação com o tipo de alimentação. Entretanto, este trabalho é de uma época em que as orientações sobre aleitamento materno e cuidados de maternagem diferiam das orientações atuais.

A associação encontrada entre desmame e o desfecho pode ser decorrente de alergia ao leite de vaca ou má absorção de carboidratos, que resulta na produção de gases, como dióxido de carbono e hidrogênio, e podem ocasionar a cólica. Ou seja, se aceita um caráter mais intrínseco para a etiologia da cólica, como descrito recentemente na metaanálise de Garrison e por outros autores $3,5,8-10,25$.

Talvez houvesse a necessidade de se avaliar melhor o temperamento materno e a assistência prestada ao lactente, uma vez que há na literatura a descrição da mãe da criança com cólica como uma mulher depressiva, cansada, com poucas respostas positivas ao cuidar da criança e uma inadequada assistência materna, e essas características poderiam dificultar a amamentação $0^{7,27}$.

A associação entre cólica e o desmame precoce nos remete a uma outra idéia relacionada ao problema, que a cólica é um dos principais motivos do oferecimento de chás para lactentes, tanto por parte das famílias como pelos médicos ${ }^{26,28}$. Sabe-se também que a introdução dessa prática é fator de risco importante para o desmame precoce, e para que essa conduta seja efetiva, é necessária a ingestão de grandes volumes ${ }^{10}$, o que pode comprometer seriamente a nutrição da criança. A introdução de chás está também associada com quadros diarréicos. Esses fatores aumentam o risco de morbimortalidade nessa faixa etária ${ }^{29}$. Portanto, julga-se importante enfatizar o papel protetor do aleitamento materno para o problema, e o cuidado que se deve ter ao se confirmar o diagnóstico materno de cólica.

\section{Referências bibliográficas}

1. Wessel MA, Cobb JC, Jackson EB, Harris GS, Detwiler AC. Paroxysmal fussing in infancy, sometimes called "colic". Pediatrics 1954;14:421-34.

2. Fractman MSV. Las perturbaciones funcionales del lactante. Revista del Hospital de Niños 1982;24:99-102.

3. Balon AJ. Management of infantile colic. Am Family Physician 1997;56:235-42.
4. Leger DW, Thompson RA, Merrit J, Benz J. Adult perception of emotion intensity in human infant cries: effects of infant age and cry acoustics. Child Dev 1996;67:3238-49.

5. Geertsma A. Cólicas - uma síndrome dolorosa do lactente? Clin Ped Am Norte 1989;4:955-71.

6. Field PA. A comparison of symptoms used by mothers and nurses to identify an infant with colic. Int J Nurs Stud 1994;31:201-15.

7. Baar RG, Rotman A, Yaremko J, Leduc D, Francouer TE. The crying of infants with colic: a controlled empirical description. Pediatrics 1992;90:14-21.

8. Cheyne PS, Kulczycki A. Human breast milk contains bovine IgG. Relationship to infant colic? Pediatrics 1991;87:439-44.

9. Treem WR. Infant colic. A pediatric gastroenterologist's perspective. Pediatr Clin North Am 1994;41:1121-38.

10. Garrison MM, Christakis DA. A systematic review of treatments for infant colic. Pediatrics 2000;106:184-90.

11. Bocquet A, Bresson JL, Briend A, Chouraqui JP, Darmaun D, Dupont C, et al. Infant formulas and soy protein - based formulas: current data. Arch Pediatr 2001;8(11):1226-33.

12. Forsyth B. Colic and the effect of changing formulas: a doubleblind, multiple crossover study. J Pediatr 1989;115:521-6.

13. Baar RG. The "colic" enigma: prolonged episodes of a normal predisposition to cry. Infant Mental Health J 1990;11:340-8.

14. Jacobson D, Melvin N. A comparison of temperament and maternal bother in infants with and without colic. J Pediatr Nurs 1995;10:181-8.

15. Rautava P, Helenius H, Lehtonen L. Psychosocial predisposing factors for infantile colic. Br Med J 1993;307:600-4.

16. Canivet C, Jakobsson I, Hagander B. Infantile colic. Follow-up at four years of age: still more "emotional". Acta Paediatr 2000; 89:13-7.

17. Huhtala V, Lehtonen L, Heinonen R, Korvenranta H. Infant massage compared with crib vibrator in the treatment of colicky infants. Pediatrics 2000;105:E84-86.

18. Armstrong K, Previtera N, Mc Callum R. Medicalizing normality? Management of irritability in babies. J Paediatr Child Health 2000;36(4):301-5.

19. Lucassen PLBJ, Assendelft WJJ, van Eijk JThM, Gubbels JW, Douwes AC, van Geldrop WJ. Systematic review of the occurrence of infantile colic in the community. Arch Dis Child 2001;84: 398-403.

20. Takeda, SMP. Avaliação de unidade de atenção primária: modificação dos indicadores de saúde e qualidade da atenção [dissertação] Pelotas: Universidade Federal de Pelotas; 1993.

21. Rutter M. Associação Brasileira de Institutos de Pesquisa de Mercado (ABIPEME). Pesquisa de Mercado. São Paulo: Ed. Ática; 1988.

22. Luz JH, Severini MH, Cardoso M. Cólicas do lactente: a visão do pediatra. J Pediatr (Rio J) 1991;67:393-9.

23. Taubman B. Clinical trial of the treatment of colic by modification of parent-infant interaction. Pediatrics 1984;74:998-1003.

24. Crowcroft NS, Strachan DP. The social origins of infantile colic: questionnaire study covering 76,747 infants. BMJ 1997;314: 1325-33.

25. Hill DJ, Hosking CS. Infantile colic and food hypersensitivity. J Pediatr Gastroenteol Nutr 2000;30:67-76.

26. Sarinho ESC, Monte L, Couto CS, Azevedo EC, Cruz MRLR. Tipo de aleitamento x cólica. Rev IMIP 1991;5:91-4.

27. Pauli-Pott U, Becker K, Mertesacker T, Beckmann D. Infants with "colic"-mothers' perspectives on the crying problem. J Psychosom Res 2000;48:125-32. 
28. César JA, Kuhn D, Devens ES, Martins E Jr., Aguiar MRC, Holthausen RS, et al. Prescrição de chás para crianças menores de seis meses: a opinião dos médicos de uma cidade de porte médio no sul do Brasil. J Pediatr (Rio J) 1996;72:27-31.

29. Giugliani ERJ. O aleitamento materno na prática clínica. J Pediatr (Rio J) 2000;76:S238-252.
Endereço para correspondência:

Dra. Maria Amália Lutz Saavedra

Rua São Borja, 208 - Laranjal

CEP 96090-660 - Pelotas, RS

Fone: (53) 227.8488

E-mail: saavedra@terra.com.br 